Recepción: 27 / 12 / 2017

Aceptación: 16 / 02 / 2018

Publicación: 08 / 05 / 2018

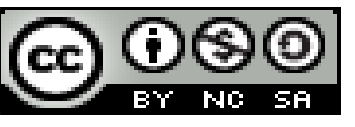

Ciencias de la salud

Artículo de Revisión

\title{
Índice de estética dental en niños en la consulta de Odontopediatría
}

\section{Dental aesthetics index in children in the pediatric dentistry office}

\section{Índice de estética dentária em crianças no consultório de odontopediatria}

\author{
Juan Antonio Oliveira-del Río ${ }^{\mathrm{I}}$ \\ juanoliveiradelrio@hotmail.com \\ Sandra Sandoval-Pedauga II \\ sandra.sandoval@gmail.com \\ Miguel Carrasco-Sierra III \\ miguel.carrasco@hotmail.com
}

Correspondencia: juanoliveiradelrio@hotmail.com

\footnotetext{
I Magister Scientiarum em Odontopediatria, Doctor en Ciencias Odontológicas, Odontólogo, Docente de la Universidad Laica "Eloy Alfaro" de Manabí, Manta, Ecuador.

II Especialista en Rehabilitación Oral, Odontóloga, Docente de la Universidad Laica "Eloy Alfaro" de Manabí, Manta, Ecuador.

III Especialista de Primer Grado en Prótesis Estomatológica, Master en Atención de Urgencias en Estomatología, Doctor en Etimología, Docente de la Universidad Laica "Eloy Alfaro" de Manabí, Manta, Ecuador.
} 


\title{
Resumen
}

Se realizó un estudio descriptivo y transversal 155 niños de 12 años de edad, que asistieron a la consulta de odontopediatria, en el período de enero del 2016 a igual mes del 2017, con la finalidad de identificar la necesidad de tratamiento ortodóncico en ellos a través del índice de estética dental. En la casuística la mayoría de los pacientes atendidos los principales componentes del índice de estética dental hallados fueron el apiñamiento (64.5\%), seguido de la irregularidad anterior (superior) 60.6\%, y el diastema (54.8\%). Se demostró que la mayor cantidad de estudiantes no necesitaban tratamiento ortodóncico, puesto que $35.4 \%$ de la serie presentaban maloclusión normal o leve.

Palabras clave: maloclusiones; estética dental; índice de estética dental; tratamiento ortodrómico.

\begin{abstract}
A descriptive and cross-sectional study was conducted of 155 children of 12 years of age, who attended the pediatric dentistry consultation, in the period from January 2016 to the same month of 2017, in order to identify the need for orthodontic treatment in them. through the dental aesthetic index. In the casuistry, most of the patients attended the main components of the aesthetic dental index found were the crowding (64.5\%), followed by the anterior irregularity (superior) $60.6 \%$, and the diastema (54.8\%). It was shown that the largest number of students did not need orthodontic treatment, since $35.4 \%$ of the series presented normal or mild malocclusion.
\end{abstract}

Keywords: malocclusions; dental aesthetics; dental aesthetic index; orthodromic treatment.

\section{Resumo}

Foi realizado um estudo descritivo e transversal com 155 crianças de 12 anos de idade, que compareceram à consulta de odontopediatria, no período de janeiro de 2016 a igual mês de 2017, a fim de identificar a necessidade de tratamento ortodôntico nas mesmas. através do índice estético dentário. Na casuística, a maioria dos pacientes atendidos nos principais componentes do índice estético dentário encontrado foi o apinhamento (64,5\%), seguido da irregularidade anterior (superior) 60,6\% e do diastema (54,8\%). Foi demonstrado que o maior número de estudantes não necessitou de tratamento ortodôntico, já que 35,4\% das séries apresentaram má oclusão normal ou leve. 
Palavras chave: maloclusões; estética dentária; índice estético dentário; tratamento ortodrômico.

\section{Introducción}

La creciente importancia de la apariencia y estética dentaria, ha aumentado la demanda de tratamiento ortodóncico a edades tempranas. ${ }^{1-2}$ Se plantea que la estética dental, como su nombre lo sugiere, abarca todo aquello relacionado con la belleza, la estética o el embellecimiento de sus dientes, en sus muy distintas formas y posibilidades. ${ }^{3}$ Esto genera que la decisión de buscar tratamiento ortodóncico esté influenciada por el deseo de verse bien, la auto percepción de la apariencia dentaria, la autoestima, el género, la edad y las normas sociales. ${ }^{3}$

Al respecto, la estética facial y dental óptima es la meta del tratamiento ortodóncico, pero para alcanzar este resultado se deben conocer las características consideradas como normales y agradables en los arcos dentales y las sonrisas 4

Al considerar estos factores se justifica contar con una herramienta epidemiológica capaz de determinar la necesidad de tratamiento y de estética dentaria en una escala socialmente aceptable. ${ }^{1}$ El Índice de Estética Dental (IED) es una herramienta que permite identificar la necesidad de tratamiento, priorizarla de acuerdo a aspectos objetivos y subjetivos y por tanto, permite un mejor uso de los limitados recursos disponible. ${ }^{1}$ Por otra parte, el IED ha sido adoptado por la Organización Mundial de la Salud como un índice transversal y aplicable entre diferentes grupos étnicos sin modificaciones. ${ }^{1}$ Fue desarrollado para este propósito en Iowa, Estados Unidos en $1986,{ }^{5-6}$ consiste en la medida de diez rasgos intraorales cada uno de los cuales se multiplica por un coeficiente de regresión. Estos rasgos son: dientes visibles perdidos, apiñamiento, separación, diastema, la máxima irregularidad maxilar anterior, la máxima irregularidad mandibular anterior, la superposición anterior del maxilar superior, la superposición Interior de la mandíbula, mordida abierta anterior vertical y la relación molar anteroposterior. El producto de cada medida se suma entre sí y con una constante, resultando en el puntaje final del IED. El puntaje del IED de 36 sirve como corte para diferenciar la maloclusión no discapacitante de la que si lo es. ${ }^{7-6}$

El incremento de profesionales y la evolución en las normas socioculturales sobre el concepto de oclusión aceptable, unido al aumento en la demanda de tratamientos ortodrómicos, han llevado a explorar las necesidades asistenciales reales de la población, a través de estudios epidemiológicos 
sobre la prevalencia y gravedad de las maloclusiones y la necesidad de tratamiento ortodóncico para corregirlas. ${ }^{8}$

Entre los métodos más usados para determinar las prioridades ortodoncias se encuentran los cuantitativos, o índices epidemiológicos, los cuales se han ido desarrollando con el transcurso del tiempo. 9

Considerando lo expuesto anteriormente, además del desconocimiento existente acerca de si los niños o adolescentes y sus padres perciben la necesidad de tratamiento ortodóncico o no, se decidió llevar a cabo el presente estudio.

\section{Metodología}

Se realizó un estudio descriptivo y transversal en niños de 12 años de edad, que asistieron a la consulta de odontopediatria, en el período de enero del 2016 a igual mes del 2017, con la finalidad de identificar la necesidad de tratamiento ortodóncico en ellos a través del índice de estética dental.

El universo estuvo constituido por los 155 niños (de ambos sexos), con dentición permanente y algún tipo de maloclusión, que nunca hubieran recibido tratamiento ortodóncico, quienes además dieron su consentimiento para participar en la investigación.

Se tuvieron en cuenta las variables: edad, sexo y estado oclusal; este último se determinó por el índice de estética dental, que cumple los requisitos esenciales de un índice oclusal y está dado por una ecuación de regresión estándar que incluye 10 componentes o características oclusales con sus correspondientes coeficientes, cuyos valores exactos y redondeados se muestran a continuación:

\begin{tabular}{|l|c|c|}
\hline Componente & Coheficiente & Redondeados \\
\hline Dientes visibles perdidos & 5,76 & 6 \\
\hline $\begin{array}{l}\text { Apiñamiento en los segmentos } \\
\text { incisales }\end{array}$ & 1,15 & 1 \\
\hline $\begin{array}{l}\text { Espaciamiento en los segmentos } \\
\text { incisales }\end{array}$ & 1,31 & 1 \\
\hline
\end{tabular}




\begin{tabular}{|l|c|c|}
\hline $\begin{array}{l}\text { Diastema en la línea media en } \\
\text { milímetros }\end{array}$ & 3,13 & 3 \\
\hline $\begin{array}{l}\text { Irregularidad anterior importante } \\
\text { (superior) }\end{array}$ & 6,75 & 1 \\
\hline $\begin{array}{l}\text { Irregularidad anterior importante } \\
\text { (inferior) }\end{array}$ & 1,34 & 2 \\
\hline $\begin{array}{l}\text { Resalte anterior maxilar } \\
\text { (superior) }\end{array}$ & 1,62 & 4 \\
\hline $\begin{array}{l}\text { Resalte anterior maxilar } \\
\text { (inferior) }\end{array}$ & 3,68 & 4 \\
\hline Mordida abierta vertical anterior & 3,69 & 13 \\
\hline Relación molar antero-posterior & 2,69 & 3 \\
\hline Constante & 13,39 & 13 \\
\hline
\end{tabular}

La necesidad de tratamiento ortodóncico se obtuvo a partir del valor del IED, que se clasificó en las siguientes categorías, en dependencia del estado oclusal individual:

\begin{tabular}{|l|l|l|}
\hline Clasificación & Clasificación & Interpretación \\
\hline Maloclusión menor & IED menor o igual que 25 & $\begin{array}{l}\text { No necesita tratamiento o } \\
\text { solo terapia menor. }\end{array}$ \\
\hline Maloclusión definida & IED entre 26 y 30 & Tratamiento electivo. \\
\hline $\begin{array}{l}\text { Maloclusión grave o } \\
\text { discapacitante }\end{array}$ & IED mayor o igual que 36 & Tratamiento prioritario. \\
\hline
\end{tabular}

\section{Resultados}

En la casuística, no o se encontraron diferencias significativas entre ambos sexos, pues las féminas representaron $53,5 \%$ y los varones $46.0 \%$.

Una vez aplicado el IED y obtenidos los valores del estado oclusal de cada paciente (tabla 1), se observó una primacía de aquellos con oclusión normal o maloclusión leve, 35.4, \% a los cuales le siguieron, en orden descendente, los que presentaban maloclusión definida, $27.0 \%$ grave $(25.8 \%)$ y solamente un $11.6 \%$ muy grave o discapacitante. 
Tabla 1. Gravedad de la maloclusión según índice estética dental.

\begin{tabular}{|c|c|c|c|}
\hline IED & Gravedad de la malaoclusion & No & $\%$ \\
\hline$\leq 25$ & Normal o leve & 55 & 35.4 \\
\hline$=26-30$ & Definida & 42 & 27.0 \\
\hline$=31-35$ & Grave & 40 & 25.8 \\
\hline$\geq 36$ & Muy grave o discapacitante & 18 & 11.6 \\
\hline Total & & 155 & 100 \\
\hline
\end{tabular}

Al emplear el índice de estética dental (tabla 2), en cuanto a la frecuencia de anomalías según los componentes del IED se halló una preeminencia del componente apiñamiento (64.5\%), seguido de la irregularidad anterior(superior) $60.6 \%$, y el diastema (54.8\%).

Tabla 2. Pacientes según componentes del índice de estética dental

\begin{tabular}{|l|c|c|}
\hline Componentes IDE & No & \% \\
\hline Dientes visibles perdidos & 16 & 10.3 \\
\hline Espaciamiento & 25 & 16.1 \\
\hline Apiñamiento & 100 & 64.5 \\
\hline Diastema & 85 & 54.8 \\
\hline Irregularidad anterior(superior) & 94 & 60.6 \\
\hline $\begin{array}{l}\text { Irregularidad anterior (inferior) } \\
\text { Resalte anterior maxilar } \\
\text { (superior) }\end{array}$ & 14 & 9.0 \\
\hline $\begin{array}{l}\text { Resalte anterior maxilar } \\
\text { (inferior) }\end{array}$ & 6 & 9.0 \\
\hline Mordida abierta vertical anterior & 42 & 3.8 \\
\hline Relación molar anteroposterior & 27 & 17.4 \\
\hline Total & 155 & 100 \\
\hline
\end{tabular}


Del total de la serie, $65.8 \%$ de los padres manifestaron estar satisfechos con la posición de los dientes de sus hijos y $14.1 \%$ se mostraron insatisfechos; el $87.7 \%$ no necesitan tratamiento (tabla 3).

Tabla 3. Percepción y motivación en los padres en cuanto al tratamiento ortodoncico.

\begin{tabular}{|l|c|c|}
\hline $\begin{array}{l}\text { Percepción y motivación } \\
\text { de los padres }\end{array}$ & No & \% \\
\hline Satisfechos & 102 & 65.8 \\
\hline Insatisfechos & 22 & 14.1 \\
\hline Necesitan tratamiento & 19 & 12.2 \\
\hline No necesitan tratamiento & 136 & 87.7 \\
\hline
\end{tabular}

\section{Discusión}

El uso del índice de estética dental (DAI) establece una lista de rasgos o condiciones oclusales en categorías, ordenadas en una escala de grados que permite observar la severidad de las maloclusiones, lo que hace esta condición reproducible y nos orienta en función de las necesidades con respecto al tratamiento ortodóncico de la población. ${ }^{10}$

Una variable es una característica susceptible de ser medida en las unidades de análisis que se estudian, que toma diferentes valores o grados de intensidad, en dependencia de cuál sea la unidad medida. ${ }^{11} \mathrm{El}$ sexo es una variable que comprende características peculiares. Por otra parte, la resistencia a las maloclusiones o no, imprime estéticas distintivas y estilos de vida diferenciados, donde la edad como variable biológica constituye un riesgo en dependencia del grupo poblacional y de su nivel de respuesta óptima o disminuida ante ciertas enfermedades. ${ }^{12}$

Con respecto al sexo específicamente, las cifras de esta serie resultaron similares a las de otra investigación realizada por Alemán Estévez et al, ${ }^{13}$ quienes obtuvieron 50,2\% de féminas y 49,8\% de varones; de manera semejante fueron los resultados de la investigación de Serra Pérez, L. et al ${ }^{12}$

El índice de estética dental, como herramienta epidemiológica, es importante para identificar y priorizar los problemas de la oclusión, donde se relaciona la gravedad de la maloclusión con la 
necesidad de tratamiento ortodrómico. Los resultados de este estudio difirieron de los de otras series en cuanto a la gravedad de las alteraciones en la oclusión. Pérez et al ${ }^{10}$ expusieron que 64,3\% de su muestra presentaba una maloclusión definida, con diferencias significativas entre hombres y mujeres, mientras que Fernández Pérez et $\mathrm{al}^{9}$ obtuvieron un predominio de maloclusión muy grave $(29,6 \%)$, con necesidad de tratamiento prioritario y obligatorio, al igual que Pino Román et al, ${ }^{14}$ quienes encontraron 58,2\% de adolescentes con alteraciones muy graves. Sin embargo, la investigación realizada por Navarro Nápoles $\mathrm{J}$ et ${ }^{15}$ coincide con nuestro resultado.

Boronat Catalá et al ${ }^{16}$ evaluaron la oclusión dental en niños de 9 años a través de dos índices: DAI e IOTN (siglas en inglés del índice de necesidad de tratamiento ortodóncico), y observaron que $48,8 \%$ necesitaba tratamiento electivo.

Una de las principales limitaciones de los índices epidemiológicos es que no incluyen la percepción de la maloclusión desde el punto de vista del paciente, en función de su grado de satisfacción, para así lograr el bienestar de las personas, es decir, aumentar su calidad de vida. ${ }^{15}$

No obstante, con el índice de estética dental se puede evaluar la percepción y motivación del paciente respecto a su maloclusión. Este permite conocer el estadio clínico de las alteraciones bucodentales en cada individuo y posibilita el establecer comparaciones. ${ }^{15}$

Pudo afirmarse que el análisis de los 10 componentes o características oclusales que integran el IED, permitió definir la frecuencia de anomalías que condicionan la aparición de maloclusiones, donde el apiñamiento en los segmentos incisales, el espaciamiento y el diastema resultaron predominantes, y al mismo tiempo posibilitó comprobar la multicausalidad al establecer comparaciones con otros estudios. Igualmente se identificaron las necesidades de tratamiento ortodóncico y se concluyó que la mayoría de los pacientes no necesitaban tratamiento.

\section{Referencias Bibliográficas}

1. Bernabé E, Flores-Mir C. Orthodontic treatment need in Peruvian young adults evaluated through dental aesthetic index. Angle Orthod 2006; 76(3):417-421.

2. Hamamci N, Başaran G, Uysal E. Dental Aesthetic Index scores and perception of personal dental appearance among Turkish university students. Eur J Orthod 2009; 31(2):168-73. 
3. Martínez Chávez BS. Manual de ortodoncia I. Oaxaca: Universidad Autónoma Benito Juárez; 2011.

4. Casas A, Bayona G. Estética en ortodoncia. Aesthetics in orthodontics. Rev. Estomat.

2010 [citado 29 Sep 2017]; 18(2): 33-8. Disponible en: http://bibliotecadigital.univalle.edu.co/bitstream/10893/2402/1/Estetica\%20en\%20or todoncia.pdf

5. WHO. Dental Oral Health Surveys. Basic methods. Geneva, Switzerland: World Health Organization 1997; 47-52.

6. Cons N, Jenny J, Kohout F. DAI: The Dental Aesthetic Index. Iowa City, Iowa: College of Dentistry, University of Iowa; 1986.

7. Poonacha K, Deshpande S, Shigli A. Dental aesthetic index: applicability in Indian population: a retrospective study. J Indian Soc Pedod Prev Dent 2010; 28(1):13-17.

8. Pinedo Hernández S, Ayala Rojas B, Vierna Quijano JM, Carrasco Gutiérrez R. Severidad de las maloclusiones y necesidad de tratamiento ortodóncico con el Índice de Estética Dental (DAI). Oral. 2012 [citado 18 Nov 2017]; 13(42): 884-7. Disponible en: http://www.medigraphic.com/pdfs/oral/ora-2012/ora1242c.pdf

9. Fernández Pérez I, Navarro Nápoles J, Ricardo Reyes M, Martínez Ramos MR, Arza Lahens M. Aplicación del índice de estética dental en estudiantes de la Secundaria Básica "Alberto Fernández Montes de Oca". MEDISAN. 2015 [citado 18 Nov 2017]; 19(12). Disponible en: http://scielo.sld.cu/pdf/san/v19n12/san021912.pdf

10. Pérez M, Neira A, Alfaro J, Aguilera J, Alvear P, Fierro Monti C. Necesidad de tratamiento ortodóncico según el Índice de Estética Dental en adolescentes de 12 años. [citado 18 Nov 2017]. Disponible en: aprendeenlinea.udea.edu.co/revistas/index.php/odont/article/.../15437/4511

11. Bayarre Vea. H, Hosford Saing R. Métodos y Técnicas Aplicadas a la Investigaciónen Atención Primaria de Salud. www.sld.cu/.../metodos_y_tecnicas_aplicadas_a_la_investigacion_en_atencion_prima 
12. Serra Pérez,L Castañeda Deroncelé, Ricardo Reyes,M Berenguer Gouarnaluses M y M

Comas Mirabent R. Índice de estética dental en adolescentes de una secundaria básica urbana. Rev Medisan 2016; 20(9):3047

13. Alemán Estévez MG, Martínez Brito I, Pérez Lauzurique A. Necesidad de tratamiento ortodóncico en escolares. Aplicación del índice DAI. Rev Méd Electrón. 2011 [citado 29 Sep 2017]; 33(4). Disponible en:http://www.revmatanzas.sld.cu/revista\%20medica/ano\%202011/vol4\%202011/tema 06.htm

14. Pino Román IM, Veliz Concepción OL, García Vega PA. Maloclusiones, según el índice de estética dental, en estudiantes de séptimo grado de Santa Clara. Medicent Electrón. 2014 [citado 18 Nov 2017]; 18(4). Disponible en: http://scielo.sld.cu/scielo.php?script=sci_arttext\&pid=S1029-30432014000400007

15. Navarro Nápoles J, Ricardo Reyes M, Martínez Ramos MR, López Díaz MA, Cobas Pérez N. Percepción de necesidad de tratamiento de maloclusión a través del Índice de Estética Dental. MEDISAN 2018 [citado 2017 May 12]; 22(1): [aprox. 0 p.]. Disponible en: http://www.medisan.sld.cu/index.php/san/article/view/1898

16. Boronat Catalá M, Bellot Arcís C, Montiel Company JM, Catalá Pizarro M, Almarich Silla JM. Necesidad de tratamiento ortodóncico según los índices Dental Aesthetic Index e Index of Orthodontic Treatment Need en niños de 9 años de Valencia. Rev Esp Ortod. 2015; 45(1): 712. 